\title{
Basal anti-inflammatory responses in cultured endothelial cells exposed to two conjugated linoleic acids
}

\author{
Carina Valenzuela ${ }^{1,2}$, Elizabeth Miles ${ }^{1}$ and Philip Calder ${ }^{1}$ \\ ${ }^{1}$ University of Southampton, Southampton, United Kingdom. and \\ ${ }^{2}$ Universidad de Valparaiso, Valparaiso, Chile
}

\section{Abstract}

Conjugated linoleic acid (CLA) isomers have been shown to possess anti-atherosclerotic properties, which may be related to the downregulation of inflammatory pathways. Whether low concentrations of CLAs are able to affect basal, unstimulated endothelial cell (EC) responses is not clear. The aim of this study was to evaluate the effects of two CLAs (cis-9, trans-11 and trans-10, cis-12) on basal inflammatory responses by ECs.

EA.hy926 cells (HUVEC lineage) were cultured under standard conditions and exposed to CLAs (1 and $10 \mu \mathrm{M})$ for 48 hours. MTT assay was performed to determine cell viability; incorporation of FA was confirmed by gas chromatography; inflammatory mediators were assessed by multiplex immunoassay; the relative expression of genes encoding transcription factors and inflammatory cytokines was assessed through real-time PCR and static adhesion assay was used to evaluate monocyte attachment to the EC monolayer.

CLAs were incorporated into ECs in a dose-dependent manner. Pre-treatment with CLA9,11 (1 uM) significantly reduced unstimulated, basal concentrations of MCP-1 $(\mathrm{p}<0.05)$, and CLA10,12 at $10 \mathrm{uM}$ had the same effect $(\mathrm{p}<0.05)$. Both CLAs at 10 uM increased the relative expression of $\operatorname{NF\kappa } \beta(p<0.01$ and $p<0.05$, respectively), while decreasing the relative expression of PPAR $\alpha$ $(\mathrm{p}<0.0001)$, COX-2 ( $<<0.0001)$ and IL-6 ( $<<0.0001)$. In contrast, no effect was observed in the adhesion assay for either CLA.

These results suggest that both CLAs at a low concentration have a neutral or modest anti-inflammatory effect in basal conditions, which may influence endothelial function and risk of vascular disease. Interestingly, at these low CLA concentrations some pro-inflammatory genes are upregulated while others are down regulated. This suggests complex effects of CLAs on inflammatory pathways.

\section{Conflict of Interest}

There is no conflict of interest 